\title{
Dermatologic manifestations of granulomatosis with polyangiitis: A case report and literature review
}

\author{
Ashley Thomas ${ }^{1}$, Nithya Krishnan ${ }^{1}$, Nicole Vesely ${ }^{2}$, Myint Thway ${ }^{3}$, Rafik Jacob*4 \\ ${ }^{1}$ Department of Internal Medicine, University of Florida College of Medicine Jacksonville, Florida, United States \\ ${ }^{2}$ University of Florida College of Medicine Gainesville, Florida, United States \\ ${ }^{3}$ Department of Medicine, Division of Rheumatology and Clinical Immunology, University of Florida College of Medicine \\ Jacksonville, Florida, United States \\ ${ }^{4}$ Department of Medicine, Division on General Internal Medicine University of Florida College of Medicine, Jacksonville, Florida, \\ United States
}

Received: September 4, 2019

DOI: $10.5430 /$ crim.v6n4p19
Accepted: November 11, 2019 Online Published: November 18, 2019

URL: https://doi.org/10.5430/crim.v6n4p19

\begin{abstract}
Granulomatosis with polyangiitis (GPA) is an ANCA-associated, systemic vasculitis of small and medium-sized blood vessels. GPA causes inflammation and destruction to the vessel wall and eventual tissue and organ damage. It classically affects the tissues and vasculature of the sinuses, lungs, and kidneys. The organ damage results in epistaxis, cough, hemoptysis, shortness of breath and/or even kidney failure. Cutaneous manifestations are rare, but have been reported. We discuss the case of a 27-year-old African American female who presented with skin lesions on her bilateral forearms and hands, several years after diagnosis and treatment for GPA. The lesions were isolated papules, located on bilateral elbows, palms, and the lateral edge of the 3rd digit on her left hand. The lesions were intensely pruritic and non-painful. A skin biopsy of the lesions showed neutrophilic and granulomatous inflammation with nuclear debris, a largely non-specific histopathologic finding. An extensive autoimmune work up revealed elevated c-ANCA/PR3-ANCA levels supporting a diagnosis of cutaneous GPA. GPA is diagnosed by a combination of clinical signs and symptoms, serologic testing, and histology from biopsy of affected organs. Patient's who meet the criteria should be tested for anti-neutrophil cytoplasmic antibody (ANCA), specifically c-ANCA/PR3-ANCA. A positive ANCA is supportive for GPA diagnosis. However, a negative ANCA does not rule out disease. Patients with cutaneous findings suggestive of GPA and positive c-ANCA/PR3-ANCA serologic testing should be closely followed up, which will lead to overall better prognosis, improved health outcomes and reduced patient and health care expenses.
\end{abstract}

Key Words: Granulomatosis with polyangiitis, Wegeners granulomatosis, Papules, Vasculitis

\section{BACKGROUND}

Granulomatosis with Polyangiitis (GPA), formerly known as Wegner's disease, is a small-medium vessel vasculitis. Initial signs of presentation can be very non-specific including general nasal irritiation, sinusitis or epistaxis. The organ systems most commonly involved include the respiratory tract, kidneys, skin and joints. The syndrome was first described by Dr.
Freidrich Wegner, for which the honorific name was given. ${ }^{[1]}$ However, following reports regarding involvement with Nazi scientists, the medical community was encouraged to use the descriptive terminology, Granulomatosis with Polyangiitis. GPA affects nearly 10-20 million people worldwide. There is no racial preponderance, but it is less frequently observed in African American and Japanese communities. Our case

*Correspondence: Rafik Jacob; Email: Rafik.Jacob@jax.ufl.edu; Address: 8th St. .W. University of Florida College of Medicine, Jacksonville, FL 32209 , United States. 
provides a rare exception to this pattern. ${ }^{[2]}$

\section{Case presentation}

A 27-year-old African American female with granulomatosis with polyangiitis (GPA), who was previously hospitalized for hemoptysis and respiratory failure, presented to our outpatient medicine clinic for new skin lesions on her bilateral forearms and hands. The lesions were isolated papules, located on bilateral elbows, palms, and the lateral edge of the 3rd digit on her left hand. The lesions were intensely pruritic and non-painful. The last time she was taking medication for her GPA was over a year ago when she last visited the
Rheumatology clinic. Labs were drawn and C-ANCA was elevated with a titer of 1:320 and antiproteinase-3 (PR3) antibody was elevated at $74 \mathrm{U} / \mathrm{ml}$. The biopsy of two skin lesions revealed necrotizing granulomatous dermatitis. She had been treated with cyclophosphamide, methotrexate, and rituximab in past and was, most recently, on maintenance therapy with azathioprine. Treatment with azathioprine, 100mg daily, along with a prednisone taper was started. The treatment resulted in eventual improvement in her lesions. She was referred back to Rheumatology clinic for additional management (see Figure 1).
A.

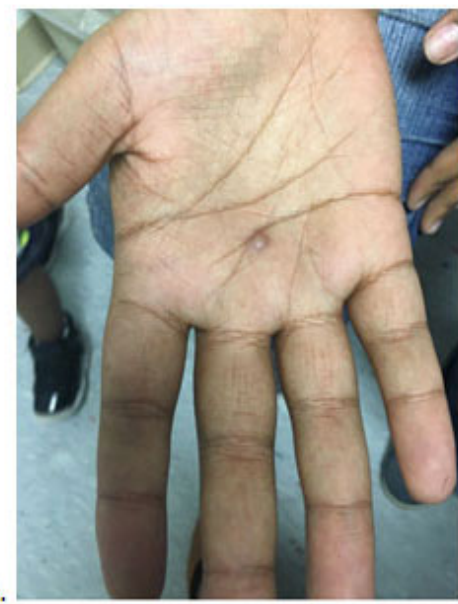

B

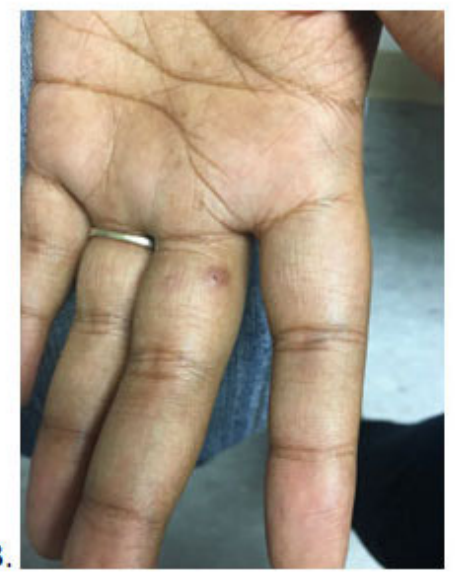

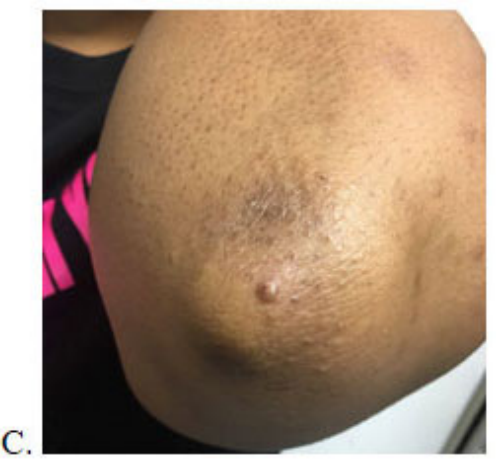

Figure 1. Single papules on bilateral palms, base of 3rd digit of left hand, and left elbow

\section{Discussion}

\subsection{Introduction}

Granulomatosis with polyangiitis (GPA), formerly called Wegener's granulomatosis, is an ANCA-associated, systemic vasculitis of small and medium-sized blood vessels. GPA causes inflammation and destruction to the vessel wall and eventual tissue and organ damage. GPA affects men and women of all ages, but more commonly occurs in individuals ages 40-60. It classically affects the tissues and vasculature of the sinuses, lungs, and kidneys. The organ damage results in epistaxis, cough, hemoptysis, shortness of breath and/or even kidney failure. GPA patients may also experience constitutional symptoms such as weight loss, fever, and fatigue.

Cutaneous manifestations are rare, but have been reported. Literature review shows anywhere from $15 \%$ to $50 \%$ of patients with GPA develop cutaneous features at some point in their disease course. ${ }^{[3]}$ In one study looking at 17 patients with GPA and cutaneous lesions, six patients had GPA with concurrent cutaneous lesions, eight patients developed skin lesions after systemic GPA findings, and three patients had cutaneous lesions preceding systemic signs of disease. ${ }^{[4]} \mathrm{Cu}$ taneous lesions can precede systemic disease anywhere from one to 10 years, or vice versa. In our case, our patient carried the diagnosis of GPA, previously manifesting as life threatening hemoptysis and respiratory failure, for many years prior to presenting with granulomatous skin lesions. She has significant long history of drug failure and irregular follow up. She presented with skin lesions only without any other signs of GPA exacerbation. Disease markers were highly elevated, along with biopsy results that were consistent with Granulomatous lesions. One interesting finding to note is, there is not one pathognomonic skin lesion or histology specific to cutaneous GPA. In this case report, we will review the various dermatologic manifestations and histopathologic findings associated with GPA, the importance of early detection and diagnosis, and overall prognosis of GPA and its treatment options.

\subsection{Diagnosis}

GPA is diagnosed by a combination of clinical signs and symptoms, serologic testing, and histology from biopsy of 
affected organs. According to the American College of Rheumatology's 1990 criteria for GPA diagnosis, patients should meet at least two out of four following criteria: ${ }^{[5]}$

(1): nasal or oral inflammation, as evidenced by painful or painless ulceration $+/$ - purulent or bloody nasal discharge

(2): abnormal chest imaging with nodules, infiltrates or cavitary lesions

(3): sediment on urine analysis showing microhematuria with $>5$ red blood cells per high power field or red blood cell casts

(4): perivascular or extravascular granulomatous inflammation on tissue biopsy

Patient's who meet the criteria should be tested for antineutrophil cytoplasmic antibody (ANCA), specifically cANCA/PR3-ANCA. A positive ANCA is supportive for GPA diagnosis. However, a negative ANCA does not rule out disease, as $20 \%$ of patient's with systemic GPA and up to $40 \%$ of limited cutaneous GPA are ANCA-negative. ${ }^{[3]}$ An attempt at tissue diagnosis should be made in all patients with active disease, as pathologic evidence clinches the diagnosis. Histopathology should reveal granulomatous inflammation. However, in the setting of cutaneous GPA lesions, there are many histologic variants.

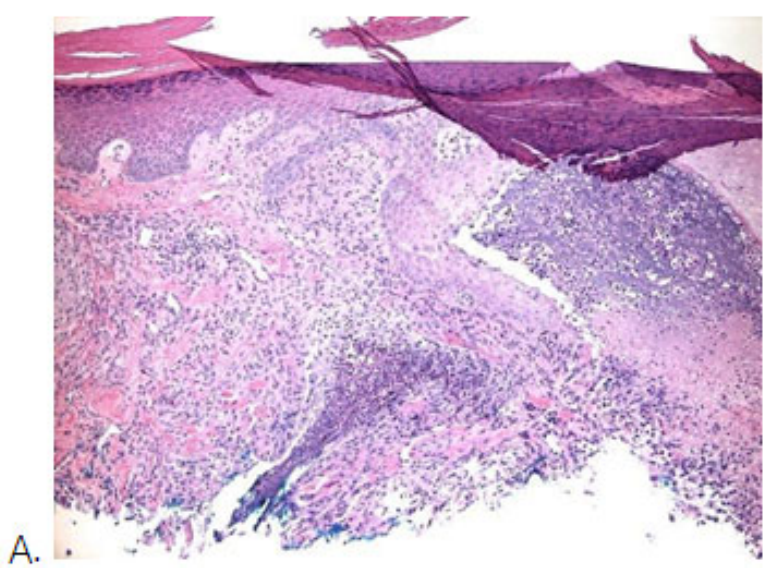

\subsection{Dermatology \& Histopathology}

There is no single dermatologic or histologic lesion that is pathognomonic for cutaneous GPA. The most common skin finding associated with cutaneous GPA is palpable. ${ }^{[6]}$ Other case reports describe papules, subcutaneous nodules similar to erythema nodosum or rheumatoid nodules, and ulcerations. Histology most commonly reveals leukocytoclastic vasculitis, but other histologic patterns include acneiform perifollicular and dermal granulomatous inflammation or palisaded neutrophilic and granulomatous inflammation. ${ }^{[5-7]}$ Elevated c-ANCA/PR3-ANCA levels further increases evidence for the diagnosis of cutaneous GPA in the setting of the aforementioned compatible histology. The table below summarizes dermatologic features in Granulomatosis with Polyangiitis compared to other cutaneous vasculidites (see Table 1). ${ }^{[7}$ Histology images from our patient are shown below (see Figure 2).

Table 1. Comparison of dermatologic manifestations of GPA and other cutaneous vasculidites

\begin{tabular}{ll}
\hline Granulomatosis with Polyangiitis & Cutaneous Small Vessel Vasculidites \\
\hline Subcutaneous nodules (granulomas) & Macules \\
Purpura & Non-blanching palpable purpura \\
Often found on Elbows & Vesicles or Shallow Ulcers \\
& Commonly noted below the waist on \\
& buttocks/thighs \\
\hline
\end{tabular}

B.

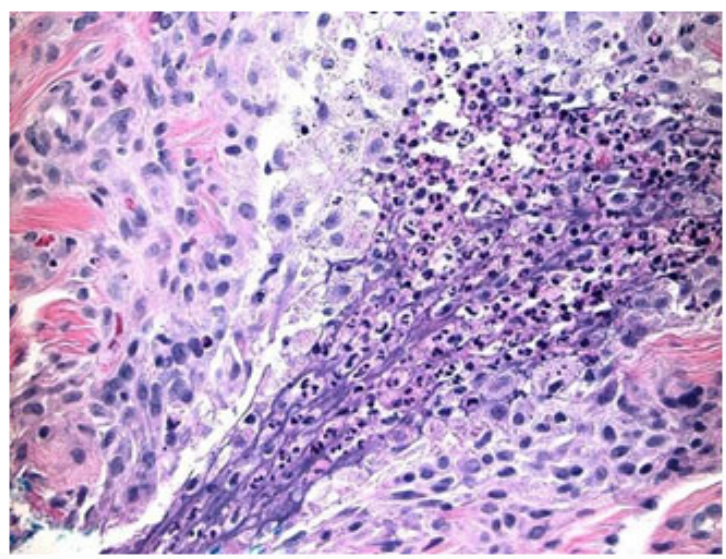

Figure 2. A. Skin biopsy showing neutrophilic and granulomatous inflammation $(H \& E, \times 5)$; B. Higher magnification, showing neutrophilic and granulomatous inflammation with nuclear debris $(\mathrm{H} \& \mathrm{E}, \times 10)$

\subsection{Treatment}

The mainstay treatment for the cutaneous manifestations of GPA is treatment of the disease itself with immunosuppressive therapy. A steroid taper can be given in the short-term for acute exacerbations and severe symptoms. However, given the well-known adverse effects of glucocorticoids, therapy should be switched to a steroid-sparing agent as soon as possible. Cyclophosphamide, a chemotherapy agent, is often used as induction therapy for the first 3-6 months of diagnosis. ${ }^{[6]}$
Given its cytotoxic effects, it is usually switched to a milder agent, like methotrexate or azathioprine, for chronic disease suppression. Complete blood count and liver function tests should be routinely monitored given the risk of myelosuppression and liver injury associated with these medications. Rituximab, a monoclonal anti-CD20 antibody, is another therapeutic option approved by the U.S. Food and Drug Administration in 2011 for the treatment of GPA. ${ }^{[4]}$ Providers should take care to screen for Hepatitis B prior to starting 
Rituximab infusions, as the drug may cause reactivation of hepatitis B virus.

\section{Conclusion}

Granulomatosis with polyangiitis, GPA, is a systemic vasculitis that most commonly affects the upper and lower respiratory tracts and kidneys. Symptoms may range from epistaxis, hemoptysis, or shortness of breath to respiratory or renal failure. Cutaneous manifestations are not commonly seen but can occur at any time in the disease course and may even be a presenting feature. The most common dermatologic lesion seen in GPA is palpable purpura and the most common histopathology seen is leukocytoclastic vasculitis. Histology in combination with ANCA serologic testing can be used to diagnosis GPA. C-ANCA and PR3-ANCA serology is highly specific and sensitive for the disease, and levels are usually elevated in systemic disease and during exacerbations, but may not be elevated in limited cutaneous disease. Patient's with cutaneous findings suggestive of GPA and positive c-ANCA/PR3-ANCA serologic testing should be closely followed up for development of systemic GPA, as early diagnosis and treatment will lead to better overall health outcomes.

\section{CONFlicts OF INTEREST Disclosure}

The author declares no conflict of interest

\section{REFERENCES}

[1] Bosch X, Guilabert A, Espinosa G, Mirapeix E. Treatment of antineutrophil cytoplasmic antibody associated vasculitis: a systematic review. JAMA. 2007; 298 (6): 655-69. PMid:17684188. https://doi.org/10.1001/jama.298.6.655

[2] Pakalniskis MG, Berg AD, Policeni BA, et al. The Many Faces of Granulomatosis With Polyangiitis: A Review of the Head and Neck Imaging Manifestations. AJR. American Journal of Roentgenology (Review). 2015 December. https ://doi .org/10.2214/AJR.14 .13864

[3] Comfere NI, Macaron NC, Gibson LE. Cutaneous manifestations of Wegener's granulomatosis: a clinicopathologic study of $17 \mathrm{pa}-$ tients and correlation to antineutrophil cytoplasmic antibody status. J Cutan Pathol. 2007; 34: 739-747. PMid:17880578. https: //doi.org/10.1111/j.1600-0560.2006.00699.x
[4] Nasir N, Ali SA, Mehmood RHM. Cutaneous Ulcers as Initial Presentation of Localized Granulomatosis with Polyangiitis: A Case Report and Review of the Literature. Case Rep Rheumatol. 2015; 5(17): 25. PMid:26664797. https://doi.org/10.1155/2015/517025

[5] Leavitt RY, Fauci AS, Bloch DA, et al. The American College of Rheumatology 1990 criteria for the classification of Wegener's granulomatosis. Arthritis Rheum. 1990; 33: 1101-1107. PMid:2202308. https://doi.org/10.1002/art.1780330807

[6] Norris MJ, Tomecki KJ, Bergfeld WF, et al. Cutaneous Wegener's granulomatosis Report of a case and review of the literature. Cleve Clin J Med. 1988; 55: 181-184. PMid:3383418. https://doi .or g/10.3949/ccjm.55.2.181

[7] Callen, Jeffrey P.; Jorizzo, Joseph L.; Zone, John J.; Piette, Warren; Rosenbach, Misha A.; Vleugels, Ruth Ann (2016-03-08). Dermatological signs of systemic disease. Callen, Jeffrey P., 1947- (Fifth ed.). Edinburgh 